\title{
De la justiciabilité des droits humains sociaux en droit international
}

\author{
Par Alfred Mwenedata, Ph. D.
}

\section{INTRODUCTION GENERALE}

Cet article se propose d'étudier le contexte des droits humains économiques, sociaux et culturels et la question de leur justiciabilité. L'on ne parlera que de la reconnaissance judiciaire des droits sociaux. Il ne s'agit pas ici de savoir si la reconnaissance des droits de l'homme sociaux les constate ou les crée ; car le problème est d'ordre spéculatif. A supposer, en effet, que la reconnaissance acquiesce à des prérogatives qui lui sont antérieures, il reste que, concrètement, les droits individuels ne sont ni invocables ni utilisables tant qu'ils ne sont pas reconnus. Depuis plusieurs décennies, la communauté internationale intensifie ses efforts pour assurer l'intégration des droits de tous un chacun sans distinction aucune dans le dispositif de protection des droits humains. En effet, l'interprétation des normes du droit international relatives aux droits humains tiennent davantage compte des rapports sociaux et juridiques qui doivent exister entre les individus eux-mêmes et entre les individus et les Etats, comme le témoignent la Charte des Nations Unies adoptée en juin $1945^{1}$ ainsi que la Déclaration universelle des droits de l'homme adoptée en décembre $1948 .^{2}$

La division entre droits civils et droits sociaux tend, de nos jours, à être remis en cause: des mouvements sociaux n'hésitent plus à revendiquer en justice le droit à la nourriture, le droit à l'eau potable, le droit à la propriété, le droit au logement ou aux soins de santé, pour ne citer que peu ; des constructions intellectuelles nouvelles s'élaborent et repensent l'universalité et l'indivisibilité des droits de l'homme ${ }^{3}$; certains juges, tant internationaux que nationaux, contribuent par leurs décisions à renforcer la justiciabilité, l'effectivité et l'opposabilité des droits sociaux. ${ }^{4}$ Ces mouvements d'évolution de la mise en exercice d'un Etat de droit social sont remarquables en Amérique du Sud, en Inde, en Afrique du Sud et se remarque également dans d'autres systèmes juridiques, notamment européens. ${ }^{5}$

Malgré le conservatisme de la primauté jadis accordée par les gouvernements occidentaux aux droits civils et politiques et la variété des objections et/ou opinions doctrinales adversaires au regard de la justiciabilité des droits sociaux, il faut combler le déséquilibre entre les droits civils et politiques et les droits économiques, sociaux et culturels pour placer les deux groupes des droits sur un pied d'égalité. Notre article vise à démontrer que les droits économiques, sociaux et culturels peuvent aussi bénéficier des mesures d'examen et des décisions de justice ; qu'il est souhaitable d'obtenir des tels jugements, et enfin qu'en pratique, des nombreux tribunaux à travers le monde ont déjà émis des tels jugements concernant plus ou moins directement les droits économiques, sociaux et culturels.

Cette série des recherches témoigne notre volonté de reconnaître l'importance d'une approche spécifique et des méthodes cohérentes pour documenter la question de la justiciabilité des droits économiques, sociaux et culturels. L'objet de la présente étude est axé sur trois grands points à savoir : Notions sur le concept des droits humains (chapitre premier), la précision du contexte des droits économiques, sociaux et culturels (chapitre 2), et le développement jurisprudentiel en matière de justiciabilité des droits sociaux (chapitre 3).

\footnotetext{
${ }^{1}$ Voir les articles 1, 55, 56 de la Charte des Nations Unies adoptée par l'Assemblée Générale des Nations Unies en 1945.

${ }^{2}$ Voir l'article 29 de la Déclaration universelle des droits de l'homme de 1945.

${ }^{3}$ A. Neier, "Social and Economic Rights: A Critique", in Human Rights Brief 13-2 (2006), 1-3.

${ }^{4}$ G. Rosenberg, The Hollow Hope: Can Courts Bring About Social Change?, Chicago: University of Chicago Press, 1991, pp 2 et ss.

5 C. Tomuschat, "An Optional Protocol for the International Covenant on Economic, Social and Cultural Rights?", in Weltinnenrecht-Liber amicorum Jost Delbrück, Duncker \& Humblot, Berlin, 2005, pp. 815-834. 


\section{Chapitre premier : Notions sur le concept des droits humains}

Dans ce chapitre relatif aux notions sur le concept des droits humains nous allons insister sur trois points à savoir : l'introduction (section première), les droits humains définis par la philosophie du droit naturel (section 2), l'universalité et relativisme culturel (section 3).

\section{Section première : Introduction}

La notion des droits fondamentaux de l'homme est aussi rarement élucidée que fréquemment utilisée. Pour Levinet :

«L'homme y signifie l'être humain au sens générique du terme. Il est vrai que les instruments juridiques internes et internationaux l'envisagent souvent réduits à une catégorie d'êtres : citoyen, femme, enfant, travailleur, étranger, réfugié, etc. En outre, ils sont parfois relatifs, non pas aux seuls individus, mais à des groupements considérés comme tels: associations (dont les formations politiques), syndicats et autres groupements à caractère professionnel, etc. Mais les difficultés de telle ou telle catégorie d'homme ne différent pas fondamentalement de problèmes soulevés par les droits de l'être humain. Toutefois, cette dernière notion s'avère floue, d'une imprécision qui est tantôt substantielle, tantôt temporelle ». ${ }^{6}$

Jusqu'à l'heure actuelle, les auteurs ne conçoivent pas de la même façon le concept des droits fondamentaux de l'homme. Certains, à l'instar de Clapham, invoquent que «les droits fondamentaux constituent une demande morale de rectifier toute sorte d'injustice dans la vie courante ; pour d'autres, ce n'est plus qu'un slogan d'être traité avec suspicion, ou bien même hostilité $»{ }^{7}$ D'autres, quelquefois, considèrent que les droits fondamentaux de l'homme représentent presqu'un terme d'art, se référant aux détails du droit national ou international des droits de l'homme, comme c'est l'idée de Jacques Mourgeon qu' «aucune description du contraire n'ayant été effectuée à cet effet, que la personne est par nature dotée des prérogatives, c'est-à-dire des facultés de consciemment faire ou agir, s'abstenir ou refuser, réclamer, obtenir et, surtout, se protéger. Par elles-mêmes, elles ne sont points des droits ${ }^{8}{ }^{8}$

Pour accéder à leur qualité, les prérogatives doivent faire l'objet d'un statut particulier : le statut juridique. Il est nécessaire, et suffisant, que la règle y touche de quelque manière : par l'acceptation, la limitation, l'organisation, la régulation, l'obligation ou l'interdiction : point de droit sans droit, et point de droit qui ne soit une prérogative, mais pas nécessairement l'inverse. Mais, si les prérogatives touchées par le droit sont constitutives des droits, ceux-ci ne sont pas tous les droits de l'homme. Les relations sociales comportent intrinsèquement l'exercice des droits qui concrétisent la sociabilité. ${ }^{9}$ Beaucoup ne sont pas directement relatifs à la personne, mais à un acte juridique tel qu'un contrat, ou à une chose : ainsi en est-il des droits du créancier à l'encontre de son débiteur, ou du propriétaire au regard de son bailleur, ou inversement. En effet, les droits fondamentaux de l'homme ne sont analysables que par la description de leur nature, et de leur contenu, en dehors de toute interprétation théorique, voire philosophique. ${ }^{10}$

Pour Alland, D., le concept des droits fondamentaux de l'homme apparait en définitive comme un concept de civilisation. ${ }^{11}$ Ainsi, l'auteur poursuit sa théorie en ces termes :

«Le concept des droits fondamentaux de l'homme indique ce qu'il y a de spécifiquement humain dans les droits des sociétés. Il peut se traduire dans diverses cultures dont la richesse doit être préservée. Son adoption n'implique pas l'uniformisation des droits positifs, mais l'intégration dans chacun d'eux des exigences d'une identité proprement humaine dont on ne peut nier l'universalité, et dont l'effectivité doit être assurée». ${ }^{12}$

D'autres chercheurs comme Leah Levin n'ont pas explicitement utilisé le terme «prérogatives », mais font recours au terme « droits moraux » :

${ }^{6}$ M. Levinet, Théorie générale des droits et libertés, Bruxelles: Bruylant, 2006, p. 163; A. Roberston et J. Merrils, Human Rights in the World, Manchester : Manchester University Press, 1996, pp.1 et ss.

${ }^{7}$ Andrew Clapham, Human Rights: a very short Introduction, Oxford : Oxford University Press, 2007, p.1; P. Gregorio, Théorie générale de droits fondamentaux, Paris : LGDJ, 2004, p. 271; R. Degni-Segui, Les droits de l'homme en Afrique noire francophone: théorie et réalités, Abidjan: Imprimob, 1997, pp. 4-5 ; Mireille Delmas-Marty, Le relatif et l'universel, Paris : Seuil, 2004, p. 3 et ss.

${ }^{8}$ Jacques Mourgeon, Les droits de l'homme, $1^{\text {ère }}$ édition, Paris : PUF, 1978, p. 6 ; F. Jullien, « Universels, les droits de l'homme?» in Le Monde Diplomatique, février 2008, p. 24.

${ }^{9}$ L. Favoreu, et al., Droit des libertés fondamentales, $3{ }^{\text {ème }}$ édition, Paris : Dalloz, 2005, p. 14.

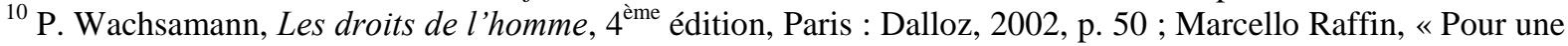
généalogie de la question des droits de l'homme » in Aspects, $\mathrm{n}^{\circ}$ 1, 2008, pp. 133-140.

${ }^{11}$ D. Alland, Droit international public, Paris : PUF, Collection Droit Fondamental, 2000, p. 577.

${ }^{12}$ Michel Villey, Le droit et les droits de l'homme, Paris : PUF, 1998, pp. 2 et ss.

DOI: 10.9790/0837-2109041329 www.iosrjournals.org $\quad 14 \mid$ Page


«Tous les êtres humains naissent égaux en dignité et en droits. Ce sont là des droits moraux inaliénables et inhérents à chaque être humain du seul fait qu'il est un être humain. Ces droits moraux sont énoncés et formulés dans ce que nous appelons aujourd'hui les droits de l'homme, et ont été traduits en droits légaux, institués conformément aux règles juridiques en vigueur dans les sociétés tant nationales qu'internationales. Ces droits ont leur fondement dans le consentement des gouvernés, c'est-à-dire des sujets des droits ». ${ }^{13}$ Les croyances anciennes des sociétés ont souvent affirmé que les droits fondamentaux de l'homme dérivent exclusivement du droit naturel.

\section{Section 2 : Droits humains définis par la philosophie du droit naturel}

Le terme «droits fondamentaux de l'homme» prend racines dans les croyances anciennes des hommes, dans ce sens que tout être humain a des devoirs et responsabilités; tout comme des interdictions à respecter. ${ }^{14}$ Dans une spéculation philosophique, le concept des droits naturels a donné une base initiale au développement de la notion des droits humains tout en imposant une opposition entre les individus et le Gouvernements : les citoyens ont des droits que les Etats doivent respecter, ${ }^{15}$ afin d'éviter la torture et la barbarie ou tout ce qui pourrait être traitement inhumain en l'encontre de ceux qui naissent libres et égaux. C'est ainsi que la Déclaration universelle des droits de l'homme de 1948 en a été le document constitutionnel, initié par les Nations Unies pour «préserver la race humaine ». ${ }^{16}$

L'idée du droit naturel a émergé dans le Moyen-âge. Elle a été développée par les grecs classiques et chrétiens de l'époque mais elle a été renforcée par la théologie catholique médiévale de ces temps-là. La tradition du droit naturel dit qu'à cause de sa nature humaine, l'homme naît pour faire le bien et non pour le mal. C'est la concrétisation des valeurs morales qui sont en lui. Ces valeurs morales ont le rôle de générer des droits et devoirs qui ne seraient pas justifiées, ni limitées par l'application d'un système juridique particulier, par la communauté, Etat, race ou civilisation. En principe tout être humain est sujet aux règles, et est capable de discerner le contenu des normes naturelles. ${ }^{17}$

Le point de départ de l'internationalisation du concept des droits de l'homme est l'impact des traumatismes qu'avait laissé derrière elle la seconde guerre mondiale. Celle-ci était un conflit unique en son genre compte tenu des souffrances qu'il en a causées. Les niveaux sans précédent de brutalité qui a caractérisé la campagne génocidaire qui a coûté des millions des vies des juifs ont prouvé que la deuxième guerre mondiale n'était pas seulement une lutte militaire à la quête de la victoire mais une nécessité de créer un nouvel ordre international qui ne permettrait plus jamais que ces horreurs se reproduisent. ${ }^{18}$ Les procès de Nuremberg sur les crimes de guerre contre les officiers nazis pendant 1945 et 1946 marquent le premier pas dans cette direction.

Aujourd'hui, la pratique commune est que les êtres humains ont des droits parce qu'ils sont humains et non parce qu'ils appartiennent à tel ou tel autre Gouvernement. ${ }^{19}$ Bien entendu, ce ne sont pas des Etats qui confèrent les droits à leurs populations, celles-ci naissent avec des prérogatives qui sont liées à leur personnalité humaine. Dans son ouvrage consacré au concept des droits humains, Christian Tomuschat écrit :

«The very idea of human rights presupposes a certain concept of the human being. By recognizing legal entitlements to every person, to men and women, to children and elderly persons, to business managers and to members of tribal communities alike, the international community has acknowledged that indeed all human beings have something in common. They are all recognized as persons whose dignity must be respected, no matter whether the individual concerned can take his/her own decisions on his/her life». ${ }^{20}$

Cela veut dire par analogie que l'idée des droits fondamentaux de l'homme englobe le concept de l'être humain. Par le fait de reconnaitre des privilèges juridiques à toute personne, aux hommes et femmes, aux enfants et personnes âgées, aux gestionnaires d'affaires et tout comme aux communautés tribales; la

\footnotetext{
${ }^{13}$ Levin Leah, Droits de l'homme: questions et réponses, Paris : Editions UNESCO, 2005, p. 17.

${ }^{14}$ Michael Haas, International Human Rights: a comprehensive Introduction, London \& New York: Routledge, 2008, p. 17.

${ }^{15}$ Ibidem.

${ }^{16}$ Laïd Zaghlami, « Droits de l'homme et développement humain : des concepts qui ne font pas le consensus », in La Tribune (Alger), 25 juin 2005, p. 1 ; Thomas Buergental, International Human Rights, $2^{\text {nd }}$ edition, Washington : West Publishing Co, 1995, pp. 29-38.

${ }^{17}$ Robert G. Patman, Universal Human Rights, London: Macmillan Press Ltd, 2000, p. 37.

${ }^{18}$ Idem, p. 1.

${ }^{19}$ Ibidem; Voyez aussi Mary Robinson, "The Universal Declaration of Human Rights : a Living Document", Australian Journal of International Affairs, Vol. 52, No. 2, juillet 1998, p. 118.

${ }^{20}$ Christian Tomuschat, Human Rights: between Idealism and Realism, New York: Oxford University Press, 2003, p. 2. 
communauté internationale a compris que tous les êtres humains ont définitivement quelque chose en commun. Ils sont admis comme des personnes dont la dignité doit être respectée, même si la personne concernée peut prendre des décisions sur sa propre vie.

L'auteur poursuit en ces termes: «Human rights are rights which a person enjoys by virtue of being human, without any supplementary condition being required $»^{21}$. De ce qui précède, il faut entendre que les droits humains sont les droits dont la personne jouit en vertu de sa qualité d'être humain, et cette jouissance n'est soumise à aucune condition supplémentaire. Signalons à ce sujet que dans la conception qui prend en compte le droit naturel, la Déclaration universelle des droits de l'homme est un texte normatif dont les auteurs ne font que constater l'existence des droits qui préexistent à leur consécration juridique (c'est-à-dire à leur reconnaissance). ${ }^{22}$ Le droit naturel qui produit les droits fondamentaux de l'homme est l'œuvre de Dieu alors que les droits de l'homme sont organisés à leur tour par les hommes (au moyen des textes).

Les perceptions variées sur les droits humains ont aussi conduit à la création des trois générations des droits à savoir : les droits civils et politiques (appelés aussi droits justiciables) qui invitent les Etats à garantir la dignité et les libertés fondamentales des leurs citoyens en s'abstenant de toute action arbitraire (action négative), comme le droit à la vie, à l'intégrité physique, au vote; ensuite les droits économiques, sociaux et culturels (en d'autres mots droits non-justiciables) qui impliquent les Etats dans les actions positives pour assurer le bien-être et bonheur des populations comme le droit à l'éducation, à l'habitat, etc. ; enfin les droits de solidarité qui sont relatifs à la solidarité nationale comme par exemple le droit à l'environnement, à l'auto-détermination, au développement. Il ya lieu de croire que les droits de solidarité n'auraient pas la raison d'être entant qu'une génération à part et qu'ils seraient rangés dans la seconde génération qui définit presque des droits semblables.

Stephen P. Marks émet une opinion dans son article ayant trait à la distinction des grandes catégories des droits humains en ces termes :

«At the theoretical level, the case is often made that civil and political rights are permanent and immutable, and are not subject to changing circumstances as they relate to the permanent nature of human beings... another justification for the distinction between categories is based on cultural or civilizational bias, the concept of the autonomous individual being characteristic of Western modernism and requiring that civil and political rights protect that autonomy, in contradistinction to other civilizations that value the group, whether the Asian values of duty to the family and kind (state), or the dictatorship of the proletariat in societies that claim to be communist, or communal values in African societies». ${ }^{23}$

Dans toutes ces catégories, les droits civils et politiques ont été traditionnellement associés entre eux et ont trouvé priorité au sein des Etats occidentaux. ${ }^{24} \mathrm{Il}$ est généralement admis que les droits civils et politiques sont de nature à être réalisés immédiatement ; alors que les droits économiques, sociaux et culturels demandent des actions progressives pour leur mise en œuvre. En outre, la mise en œuvre des droits civils et politiques coute moins cher (l'Etat a tout simplement l'obligation de s'abstenir de certaines actions, par exemple, ne pas commettre la torture), et ces droits sont justiciables alors que les droits économiques, sociaux et culturels s'avèrent comme des droits pragmatiques, qui représentent un idéal à atteindre mais qui ne peuvent pas être exigés devant les juridictions. ${ }^{25}$ Cependant, les droits économiques, sociaux et culturels ont été accordés plus de valeur dans les pays socialistes et dans les pays en voie de développement. ${ }^{26}$

Selon Stephen P. Marks:

«Civil and political rights are attributes of the human person with which the state must not interfere, and thus are best described as "freedoms from state interference » or «droits-attribut »; while economic, social and cultural rights are "claims on the state» or "droits-créance 》. Civil and political rights are "negative» insofar as they enjoin the state from interfering in the individual's freedom to do whatever is not harmful to others, whereas economic and social rights are correlative to positive duties of the state to enable the individual

\footnotetext{
${ }^{21}$ Idem, p. 3.

22 Jack Donnelly, Universal Human Rights in Theory and Practice, London: Cornell University Press, 1989, pp. 17-24; Maurice Crauston, What are Human Rights, London: Bodly Head, 1973, p. 36.

${ }^{23}$ Stephen P. Marks, "The Past and Future of the Separation of Human Rights into Categories", Maryland Journal of International Law, vol. 24, pp. 7-10.

${ }^{24}$ Idem, pp. 16 et 18.

${ }^{25}$ David Rehman, International Human Rights Law: a practical Approach, London: Longman, 2003, p. 6; article 2 (3) du Pacte international relatif aux droits civils et politiques.

${ }^{26}$ Idem, p. 7. 
to do what he or she would like to do. The former imply abstention of the state while that latter imply claims against the state for provision of services $\gg .{ }^{27}$

Somme toute, dans toutes les deux catégories, l'Etat a des actions à accomplir pour la réalisation effective de tous les droits humains, nonobstant la présence du critère de leur différenciation. Sans toutefois s'opposer catégoriquement à la justification classique liée à la catégorisation des droits, il convient de dire que le contexte actuel enseigne que l'Etat est obligé d'agir au de-là de l'abstention pour la meilleure satisfaction des droits qui relèvent de la première génération. La distinction entre «droits positifs » et «droits négatifs » utilisée pour comparer les deux catégories des droits définies en haut, ne devrait pas être confondue avec la distinction qui existe entre «liberté négative » et «liberté positive ». Ainsi nous lisons dans l'article suscité ce qui suit: «The idea of negative liberty as referring to an individual being left to act or be as he or she pleases without interference of other persons, whereas positive liberty involved either the collective self-determination of the space of freedom, as in a democratic system, or control or mastery of someone else who determines the scope of one's actions $»{ }^{28}$

Signalons que ces droits humains tels que consacrés par la Déclaration universelle de 1948 présentent des caractères communs : ils sont sacrés, absolus (excepté si une loi en autorise une exception), universels (au travers des traités et organes de contrôle), inaliénables, indivisibles, interdépendants, hors-commerce; opposables en toutes circonstances à la communauté et aux institutions du pouvoir public. ${ }^{29}$ Ainsi, de n'importe quel pays, de n'importe quelle couleur, etc., chacun dispose du droit d'être protégé sans discrimination aucune. ${ }^{30}$ Le statut de la Déclaration universelle des droits de l'homme est unique. Contrairement aux autres documents importants comme Magna Carta, la Déclaration française des droits de l'homme et la Déclaration américaine de l'indépendance; elle reconnait des droits et libertés de manière universelle sans que l'existence des diversités raciales et culturelles n'en constituent pas un obstacle.

\section{Section 3 : Universalité et relativisme culturel}

La Déclaration proclame l'unité de la race humaine et l'égalité de tous ses membres dans l'article premier : «Tous les êtres humains naissent libres et égaux en dignité et en droits. Ils sont doués de raison et de conscience et doivent agir les uns envers les autres dans un esprit de fraternité ». D'après le contenu de cette disposition, toute inégalité et discrimination quelque soit le motif, sont interdites car incompatibles avec la philosophie de la Déclaration. Notons d'ailleurs que le document confirme cette position également en son article 2. C'est la qualité de tout être humain, sans quelque qualification additionnelle que ce soit, qui attribue à chacun des droits et libertés considérées comme constitutifs de ses pré-conditions pour vivre en dignité. ${ }^{31}$ Cette approche de la DUDH a inspiré d'autres instruments relatifs à la protection des droits humains, notamment les deux pactes internationaux relatifs aux droits de l'homme adoptés par l'Assemblée générale des Nations Unies en 1966. La seule exception qui existe est la Convention internationale relative à la protection de tous les Travailleurs migrants et les membres de leurs familles qui cherche à protéger une catégorie des populations restreinte mais particulièrement vulnérable. ${ }^{32}$

Le débat sur la question de l'universalité des droits humains a divisé les opinions en droit international et l'approche universelle des droits consacrée par la Déclaration a été critiquée pour son manque de pertinence quant à l'absence des considérations des diversités culturelles dans l'adoption de ses principes normatifs. En effet, l'argument capital qui ressort des critiques dit que la variété dans les valeurs religieuses et culturelles qui caractérisent les communautés humaines prouve qu'il n'existe pas de dénominateur commun. ${ }^{33}$ La question qui se pose est celle-ci : est-il possible de généraliser les droits et les appliquer de manière égale à tous les peuples, toutes les cultures, religions, langues?

L'idée est que les droits de l'homme universels est une création de l'école de la pensée fondée sur le christianisme européen d'après la deuxième guerre mondiale. Ainsi, les droits de l'homme ont été considérés par

\footnotetext{
${ }^{27}$ Supra note 21, p. 13.

${ }^{28}$ Ibidem.

${ }^{29}$ Symonides Janusz, Human Rights: Concept and Standards, London: Ashgate Publishing Company, 2000, pp. 32-35.

${ }^{30}$ François Juillien, Supra note 5, p. 1.

${ }^{31}$ Christian Tomuschat, Supra note 18, p. 58.

32 Convention internationale relative à la protection de tous les Travailleurs migrants et les membres de leurs familles adoptée par la Résolution (GA) 45/158 du 18/12/1990.

${ }^{33}$ Idem, pp. 56 et 59. 
certains comme des droits qui traduisent la culture occidentale au moyen d'un impérialisme culturel. ${ }^{34}$ Malgré son idée unificatrice, le principe d'universalité a été un lieu d'affrontement dans les débats politiques et les disputes éthiques, particulièrement en ce qui concerne son application dans les sociétés non-occidentales.

Alors que les sociétés arabes veulent fonder leurs droits humains sur les règles coraniques « la Shari'a », les Hindu décrivent leur système de protection des droits humains comme étant traditionnel et multidimensionnel. ${ }^{35}$ Ainsi pour les asiatiques, seuls l'ordre et la discipline comptent et moins les droits et libertés. C'est le modèle appelé « confucéen » ou confucianisme. La culture africaine est bâtie sur l'hétérogénéité qui existe dans chacune des régions qui composent le continent, etc.

Par ailleurs, la nature, la catégorisation et l'ordre de priorité des droits sont mis en question. Le passage suivant indique:

«Les droits de l'homme ne sont pas un phénomène divin, visible des origines de l'histoire à sa fin. La question de la nature des droits de l'homme dépend des systèmes philosophiques, idéologiques et sociaux en diverses périodes historiques. La Déclaration universelle des droits de l'homme n'est qu'un produit de la pensée libérale-bourgeoise ou les êtres humains sont respectés en tant qu'individus atomisés et sont responsables des conditions dans les quelles ils vivent. Ils sont respectés dans le sens qu'ils ont les mêmes droits juridiques, mais les conditions nécessaires pour réaliser l'égalité juridique, l'égalité des droits économiques restent ignorées ». ${ }^{36}$

Par évidence, dans différents pays, des droits différents se verront donner la priorité par l'Etat et les citoyens. Le principe uniforme et commun est que tout le monde est sujet des droits quelque soit sa nationalité ou statut. ${ }^{37}$ Certains auteurs affirment que les droits ne peuvent jamais être créés isolement ou artificiellement en se fondant sur l'égalité universelle mais doivent être entendus comme sacrés entant que légitimité historique particulièrement dans un Etat donné. ${ }^{38}$

Nous terminons cette section en épousant l'idée de Scholler qui dit que «la théorie des droits de l'homme cherche toujours à nouveau un droit de l'homme originel, qui pourrait être en somme l'élan vital de tous les droits de l'homme. Tout porte à croire que le droit de l'homme originel moderne se manifeste dans la double constellation du droit à la vie et à l'auto-détermination $\gg .{ }^{39}$

Même si le concept des droits humains universels suscite des divergences d'opinions, la pratique courante et régulière des Etats modernes est d'appliquer les principes communs du droit international, plus ou moins pour un sentiment d'obligation juridique. Aujourd'hui, la protection des droits fondamentaux de l'homme et le plan d'action contre les abus sont devenus deux choses indissociables. Le chapitre suivant porte spécialement sur la précision du contexte des droits économiques, sociaux et culturels relativement à leur justiciabilité.

\section{Chapitre 2 : Débat sur le contexte/contenu des droits économiques, sociaux et culturels relativement à leur justiciabilité}

Nous avons vu que dans la conception initiale relative à la nature des droits humains, seuls les droits de la première génération; c'est-à-dire les droits civils et politiques sont justiciables. Les droits économiques, sociaux et culturels qui relèvent de la seconde génération des droits restent par ailleurs sous la protection normale des Gouvernements mais ne sont pas susceptibles d'être invoqués devant la justice.

34 A. H. Robertson, et J. G., Merrills, Human Rights in the World: an Introduction to the Study of the International Protection of Human Rights, $3^{\text {ème }}$ édition, Manchester: Manchester University Press, 1994, p. 222.

${ }^{35}$ Azizur Rahman, Chowdhury et Jahid H. Bhuiyan, An Introduction to International Human Rights Law, Leiden: Martinus Nijhoff Publishers, 2010, p. 6.

${ }^{36}$ X, Human Rights and Cultural Relativism: Ali Javadi's Discussion with Azar Majedi and Koorosh Modaresi, éxtrait de WPI Briefing No 13 (16 mai 2001), transcription d'une discussion en personne à la Radio, premiere publication dans International Weekly No. 53 (11 mai 2001), tiré sur http://bataillesocialiste.worldpress.com, consulté le 2 février 2011.

${ }^{37}$ Smith et K. M. Rhona, International Human Rights: Texts and Materials, London: Routledge \& Cavendish, 2007, p. 40.

${ }^{38}$ Edmund Burke, cité par Catarina Krause et Martin Scheinin, International Protection of Human Rights : a Textbook, Jyväskylä (Finlande): Abo Akademi University Institute For Human Rights, 2009, p. 3; Burton M. Leiser et Tom D. Campbell, Human Rights in Philosophy and Practice, London: Ashgate Publishing Company, 2001, p. 87.

39 Heinrich Scholler, L'universalité de droits de l'homme, http://www.horizons_et_debats.ch, consulté le 2 février 2011. 
Le mot «justiciabilité» signifie que les personnes qui se considèrent victimes de violations de ces droits peuvent porter plainte devant un organe indépendant et impartial, demander une réparation appropriée, et, si la violation est avérée ou risque de se produire, obtenir que le recours soit effectif. ${ }^{40}$ En d'autres mots «justiciabilité» veut dire la capacité de recourir à un organe indépendant et impartial quand un droit a été violé ou risque d'être violé. Il signifie la possibilité d'accéder à un mécanisme qui garantit les droits reconnus. Un droit invocable accorde à son titulaire un moyen de recourir à une procédure légale pour le faire respecter, chaque fois que le devoir qui en découle n'est pas accompli. L'existence d'un recours judiciaire, compris à la fois comme l'accès à un tribunal approprié quand une violation a été commise ou est imminente et l'octroi d'une réparation à la victime, est l'une des caractéristiques d'un droit véritable. ${ }^{41}$

La justiciabilité d'un droit ne veut pas dire reconnaissance du bien-fondé de toute plainte déposée à ce sujet. Elle indique plutôt la possibilité d'obtenir qu'un organe indépendant et impartial examine une violation alléguée d'un droit. ${ }^{42}$ En d'autres termes, elle exige qu'une telle plainte ne soit pas exclue a priori. Le résultat final d'un procès dépend du fond de l'affaire et, le cas échant, des preuves administrées. ${ }^{43}$ Même si un organe indépendant et impartial déclare une plainte recevable, il peut décider que le plaignant a tort du point de vue soit du droit, soit des faits. ${ }^{44}$ La plupart des critiques sur la justiciabilité des droits sociaux sont basées sur le caractère vague ou imprécis de ceux-ci comme les lignes qui suivent le décrivent.

\section{Section unique : Arguments sur la nature justiciable des droits économiques, sociaux et culturels}

Les adversaires de la justiciabilité des droits économiques, sociaux et culturels affirment qu'ils sont si flous ou vagues qu'il n'est pas possible d'en définir suffisamment le contenu, d'où l'impossibilité de les faire valoir devant des tribunaux. ${ }^{45}$ Selon cette opinion, tandis que les droits civils et politiques indiquent clairement les conditions à remplir pour les respecter, les droits économiques, sociaux et culturels ne fixent que des objectifs souhaitables et théoriques; leur contenu varie et manque de l'exactitude nécessaire pour servir de base à une décision judiciaire.

Cependant, la question du contenu et de la portée d'un droit ne se pose pas uniquement pour les droits économiques, sociaux et culturels. On peut considérer que le contenu de tout droit n'est pas assez précis, que ce droit soit qualifié de «civil», «politique», «social», «économique» ou «culturel». En effet, de nombreuses règles légales s'expriment dans un sens large et, jusqu'à un certain point, en termes forcément généraux. ${ }^{46}$

Dans le but de déterminer le contenu et la portée des droits économiques, sociaux et culturels, la Commission Internationale des Juristes (CIJ) énonce les principes suivants dans sa Déclaration et son Plan d'action de Bangalore:

«Une définition plus précise des aspects des droits économiques, sociaux et culturels qui sont plus facilement applicables en justice exige des compétences juridiques et de l'imagination. Il est nécessaire de définir les obligations juridiques avec précision, d'indiquer clairement ce qui constitue une violation, de préciser les conditions régissant le traitement des plaintes, d'élaborer des stratégies pour lutter contre les abus et les

${ }^{40}$ Commission Internationale des Juristes (CIJ), Les tribunaux et l'application des droits économiques, sociaux et culturels : étude comparative d'expériences en matière de justiciabilité, série des droits de l'homme et état de droit, No 2, Genève, 2010, p. 1.

${ }^{41}$ Idem, p. 7 ; Par exemple, selon l'opinion émise après la Première Guerre mondiale dans l'affaire du Lusitania et considérée souvent comme fondamentale dans la définition de la responsabilité de l'Etat, «Le droit civil et la «common law » reconnaissent tous deux que toute «atteinte à un droit privé» implique un dommage et en prévoient la réparation.», Commission mixte de réclamations germano-américaine, 1er novembre 1923, Recueil de sentences arbitrales, Volume VII, p. 32. Pour un exposé global sur le sujet, voir le Guide pratique $n^{\circ} 2$ de la Commission internationale de juristes intitulé Le droit à un recours et à obtenir réparation en cas de violations graves des droits de l'homme (CIJ, Genève 2006, disponible en anglais, français et espagnol).

${ }^{42}$ K., VASAK, (1976): Le droit international des droits de l'homme. Recueil des cours de l'Académie de droit international, 1974-IV. La Haye, A.W.Sijthoff, Leyde ; pp. 388-389.

${ }^{43}$ Ibidem.

${ }^{44}$ Voir supra note 38, p. 10.

45 Voir H. L. A. Hart, The Concept of Law (Oxford: Clarendon Press, 1961; $2^{\mathrm{e}}$ édition complétée par un postscriptum de J. Raz \& P. Bulloch (eds.), Oxford: Oxford University Press, 1994, Chapitre VII); G. Carrió, Notas sobre derecho y lenguaje (Buenos-Aires: Abeledo-Perrot, 1964) 45-60; I. Trujillo Pérez, «La questione dei diritti sociali», in Ragion Pratica 14, 2000, p. 50.

${ }^{46}$ Ibidem. 
manquements et de proposer des voies de droit, lorsque cela s'impose, pour permettre la réalisation des objectifs jugés souhaitables». ${ }^{47}$

Paradoxalement, la conséquence de cette conviction visqueuse que les droits économiques, sociaux et culturels ne sont pas justiciables a été l'absence, de la part du pouvoir judiciaire des nombreux pays, de tout effort pour les combler. Parce qu'on les considère purement comme des bonnes intentions, parce que les juristes et les théoriciens du droit ne cherchent pas à les interpréter, il existe peu de concepts aidant à comprendre le droit à l'éducation, le droit à la santé, le droit à un logement convenable, le droit à l'alimentation, pour ne citer que ceux-ci. Néanmoins, le manque d'explication pratique concernant plusieurs de ces droits ne permet pas de prétendre qu'il n'est nullement possible de les définir, en tant que catégorie, à cause de certains traits essentiels ou cachés. On n'a guère tenté de définir le contenu de ces droits, puisque les critiques affirment que ce serait peine perdue. Cette absence de données pratiques est alors utilisée comme argument pour prouver que ces droits ne sont pas justiciables. ${ }^{48}$

L'article 2 du Pacte international relatif aux droits économiques, sociaux et culturels semble d'emblée minimiser la portée juridique des droits sociaux. Selon la teneur de cet article, «les Etats parties devraient prendre des mesures au maximum de leurs ressources disponibles en vue d'assurer progressivement le plein exercice des droits reconnus dans le présent Pacte. Afin d'atteindre ce but, les Etats parties useront de tous les moyens appropriés, y compris en particulier l'adoption de mesures législatives». ${ }^{49}$

Il faut déduire de cette clause le caractère purement programmatique des droits sociaux. Concrètement, cela signifierait le refus de les considérer comme des droits individuels et justiciables, mais seulement comme des buts que les Etats parties poursuivent à long terme, dans la mesure des ressources disponibles. ${ }^{50}$ Cette interprétation est peu à peu remplacée par une lecture plus contraignante. Comme pour les droits civils et politiques, les droits économiques, sociaux et culturels obligent les Etats parties à prendre des mesures précises afin d'assurer le respect des droits sociaux contenus dans le Pacte.

On reconnaît de plus en plus que les droits individuels classiques se différencient seulement, de manière graduelle, de droits économiques, sociaux et culturels. Selon une classification devenue courante, les droits humains impliquent trois types d'obligations pour les Etats parties: obligation de respecter, obligation de protéger, obligation de garantir et de donner effet. Respectivement cela signifie que l'Etat doit respecter les droits humains des individus; ensuite l'Etat doit empêcher des tiers de porter atteinte aux droits humains des individus; et enfin, l'Etat doit prendre les dispositions nécessaires afin de garantir la réalisation des droits humains.

Les Etats parties sont donc obligés de respecter les droits sociaux. Sous ce premier aspect, les droits sociaux sont considérés comme directement justiciables, c'est-à-dire qu'ils sont de nature à pouvoir être invoqués par une personne ou un groupe de personnes lors d'une procédure judiciaire ou quasi-judiciaire. Par exemple, certains groupes des personnes exclues du système d'enseignement primaire pourraient faire valoir l'obligation de l'Etat de respecter leur droit à l'éducation. ${ }^{51}$ Les Etats parties sont obligés de protéger les droits sociaux. Sous ce deuxième aspect, les droits sont également justiciables. A titre d'exemple, les enfants doivent être protégés dans leur droit de recevoir un enseignement de base (notamment contre leurs parents, si ceux-ci souhaiteraient les en empêcher). ${ }^{52}$

En 1948, le préambule de la Déclaration Universelle des droits de l'homme faisait de l'abolition de la pauvreté un des objectifs premiers de la fondation des Nations Unies. ${ }^{53}$ Soulignant l'importance accordée à la protection contre la misère, la Déclaration universelle, en son article 22 reconnaissait pour la première fois des droits économiques et sociaux à égalité avec les droits civils et politiques:

«Toute personne, en tant que membre de la société, a droit à la sécurité sociale; elle est fondée à obtenir la satisfaction des droits économiques, sociaux et culturels indispensables à sa dignité et au libre développement

\footnotetext{
${ }^{47}$ Déclaration et Plan d'action de Bangalore, $\S 18,2,1995$.

${ }^{48}$ Ibidem.

49 Voir le Commentaire général N. 3 du Comité des droits économiques, sociaux et culturels (1990) à ce sujet.

50 Voir le Commentaire général N. 9 du Comité des droits économiques, sociaux et culturels (1998).

${ }^{51}$ Article 13 du Pacte international relative aux droits économiques, sociaux et culturels (1966).

${ }^{52}$ Préambule de la Déclaration universelle de droits de l'homme de 1948 ; Article 29 (1) (b) de la Convention relative aux droits de l'enfant de 1989.

${ }^{53}$ Le Préambule de la Déclaration universelle des droits de l'homme de 1948 déclare : «... Considérant que dans la Charte les peuples des Nations Unies ont proclamé à nouveau leur foi dans les droits fondamentaux de l'homme, dans la dignité et la valeur de la personne humaine, dans l'égalité des droits des hommes et des femmes, et qu'ils se sont déclarés résolus à favoriser le progrès social et à instaurer de meilleures conditions de vie dans une liberté plus grande ».
}

DOI: 10.9790/0837-2109041329 www.iosrjournals.org $\quad$ 20|Page


de sa personnalité, grâce à l'effort national et à la coopération internationale, compte tenu de l'organisation et des ressources de chaque pays». ${ }^{54}$

En vertu de l'article 25 de la même Déclaration:

«Toute personne a droit à un niveau de vie suffisant pour assurer sa santé, son bien-être et ceux de sa famille, notamment pour l'alimentation, l'habillement, le logement, les soins médicaux ainsi que pour les services sociaux nécessaires ; elle a droit à la sécurité en cas de chômage, de maladie, d'invalidité, de veuvage, de vieillesse ou dans les autres cas de perte de ses moyens de subsistance par suite de circonstances indépendantes de sa volonté . $^{55}$

A la lumière de ces dispositions contenues par le droit international des droits de l'homme, est-ce qu'on peut admettre aujourd'hui que les droits économiques, sociaux et culturels, autrement appelés les droits des pauvres, soient non simplement promus, protégés et respectés; mais aussi justiciables? A cette question, d'aucuns affirment qu'une évolution notable pourrait résulter de l'adoption du protocole facultatif au Pacte international des Nations Unies sur les droits économiques sociaux et culturels (PIDESC). Accueilli comme une véritable révolution par le monde des organisations non gouvernementales et syndicats en effet, ce texte est le fruit de plusieurs dizaines d'années de pressions de la société civile en faveur de la reconnaissance de la justiciabilité des droits économiques, sociaux et culturels. En introduisant en effet un mécanisme de pétition, le Protocole optionnel au PIDESC renforcerait les possibilités des millions d'exclus et des victimes des droits humains d'obtenir justice. L'idée centrale est que cette révolution (ou évolution) pourrait contribuer à ce que les droits des pauvres cessent d'être des pauvres droits. ${ }^{56}$ Dans les lignes qui suivent, nous allons principalement nous pencher sur le développement jurisprudentiel en matière de justiciabilité des droits sociaux.

\section{Chapitre 3 : Le développement juridictionnel en matière de justiciabilité des droits économiques, sociaux et culturels (DESC)}

Certains jugements rendus par les courts et tribunaux différents en rapport avec la mise en œuvre des droits fondamentaux sociaux marquent le développement majeur dans le domaine de la justiciabilité des droits économiques, sociaux et culturels comme les paragraphes suivants le montrent. Certains cas concernent notamment les droits essentiels et immédiats et les mesures liées à la réalisation progressive des droits (section première), d'autres cas ne sont relatifs qu'aux à l'approche juridictionnelle à propos de l'interdiction des mesures régressives (section 2), ainsi nous aurons d'autres cas des droits humains sociaux examinés dans la jurisprudence internationale (section 3 ).

\section{Section première : Jugements sur les devoirs essentiels et immédiats et sur les mesures liées à la réalisation progressive des droits}

A ce point, la Cour constitutionnelle fédérale et le Tribunal administratif de l'Allemagne ont décrit le «contenu essentiel minimum» en se fondant sur les principes constitutionnels de l'Etat-providence et la notion de dignité humaine. Dans ce pays, les tribunaux ont décidé que ces principes constitutionnels pouvaient se traduire en l'obligation positive de l'Etat d'assurer un minimum «vital» ou «existentiel» qui comprend l'accès des personnes dans le besoin à l'alimentation, au logement et à l'assistance sociale. ${ }^{57} \mathrm{La}$ Cour constitutionnelle fédérale allemande a élaboré la doctrine du «minimum vital», ou du «niveau minimal d'existence», (Existenzminimum). En vertu de cette doctrine, l'Etat a l'obligation de fournir une assistance aux plus démunis pour leur permettre de vivre dignement. La Cour a estimé que le parlement doit certainement, en vertu de la constitution, adopter un programme social ${ }^{58}$ et que l'assistance plus démunis est, indéniablement, une des tâches de l'Etat-providence (Sozialstaat), et que ce dernier doit donc créer les conditions minimales pour que ces personnes puissent vivre dignement. ${ }^{59}$

Selon la Cour constitutionnelle, le devoir de l'Etat d'assurer des conditions minimales qui permettent une existence appropriée se fonde sur le principe de la dignité humaine garanti par l'article 1(1) de la

\footnotetext{
${ }^{54}$ Article 22.

${ }^{55}$ Article 25.

${ }^{56}$ Pierre-Henri, IMBERT, Révolution de droit public, 1989, p. 739.

${ }^{57}$ Voir, par exemple, la Cour constitutionnelle fédérale allemande $(B V e r f G)$ et le Tribunal administratif fédéral allemand (BVerwG), BVerfGE 1,97 (104f ); BVerwGE 1,159 (161); BVerwGE 25, 23 (27); BVerfGE 40, 121 (133, 134); BVerfGE 45, 187 (229); BVerfGE. 82, 60 (85) et BverfGE 99, 246 (259; Voir, aussi, le point 9 des Principes de Maastricht.

${ }^{58}$ [BVerfGE 1, 97 (104)].

${ }^{59}$ [BVerfGE 40, $\left.121(133)\right]$. 
constitution ou loi fondamentale allemande, combiné avec le principe de l'Etat-providence protégé à l'article 20 de la constitution allemande) ${ }^{60}$. S'inspirant de cette doctrine, la Cour constitutionnelle a jugé:

que l'Etat doit s'efforcer de fournir de bonnes conditions de vie aux personnes tombées dans le besoin à cause du régime hitlérien; toutefois, une demande d'appliquer la constitution n'est recevable que si le législateur ne s'acquitte délibérément pas de cette obligation qui découle de dispositions instaurant l'Etatprovidence. $^{61}$

que l'Etat doit fournir une assistance sociale à ceux qui, à cause d'un handicap physique ou mental, éprouvent des difficultés sur le plan personnel et social et ne peuvent se débrouiller seuls ${ }^{62}$; le législateur doit mettre en place les conditions minimales permettant à ces personnes de vivre dignement; il dispose d'une certaine latitude quant à l'assistance sociale à leur accorder. ${ }^{63}$

De même, le Tribunal fédéral suisse a arrêté que les tribunaux suisses pouvaient imposer aux autorités publiques le respect d'un droit constitutionnel non écrit qui garantit des conditions minimales d'existence aux ressortissants tant étrangers que suisses. ${ }^{64}$ Les tribunaux du Brésil se sont prononcé dans le même sens quand ils ont indiqué qu'au vu de la disposition précise de la constitution nationale qui établit le droit à l'éducation, l'Etat a l'obligation d'assurer aux enfants jusqu'à six ans l'accès à une crèche ou un jardin d'enfants. La Cour suprême fédérale brésilienne estime que la mise en œuvre de cette disposition constitutionnelle ne peut être laissée au libre choix des autorités administratives. ${ }^{65}$ L'accès à des soins de santé de base est également considéré comme une composante indispensable du droit à la santé. La Cour suprême argentine a souligné que, puisque la constitution et les pactes internationaux relatifs aux droits de l'homme garantissent le droit à la santé, il faut comprendre la loi qui prévoit l'accès aux services médicaux comme obligeant le personnel soignant de fournir tous les soins essentiels en cas de besoin. ${ }^{66}$

Le paragraphe premier de l'article 2 du Pacte international relatif aux droits économiques, sociaux et culturels permet la réalisation progressive de ces droits ${ }^{67}$, que les signataires reconnaissent que, dans maintes circonstances, la réalisation des droits ne peut se faire que peu à peu. Cependant, le Comité des droits économiques, sociaux et culturels pense que la réalisation progressive ne s'applique pas à tous les devoirs et que certains de ceux-ci ont un effet immédiat. ${ }^{68}$ Par conséquent, si, pour certains droits, l'Etat dispose d'une marge de manœuvre et peut choisir le calendrier approprié et attribuer les ressources requises, selon la disponibilité de ces dernières, il est d'autres devoirs qu'il doit accomplir immédiatement. Cette distinction vaut aussi pour la justiciabilité, car elle signifie qu'un organe juridictionnel peut vérifier directement si l'Etat s'acquitte de son obligation immédiate, ce qui réfute l'idée que la mise en œuvre de tous les droits économiques, sociaux et culturels est laissée à l'appréciation des pouvoirs politiques. Les critères en matière d'examen des devoirs d'application progressive sont différents, moins rigoureux et, peut-être, moins coercitifs. La Cour constitutionnelle de la Colombie a opté clairement pour cette conception, puisqu'elle a distingué, lors de l'interprétation des droits économiques, sociaux et culturels, entre les devoirs liés directement à la protection de la vie, qui ont un effet immédiat, et les autres mesures visant la réalisation progressive de ces droits. ${ }^{69}$ Mais elle a estimé aussi que les droits dont la réalisation est progressive sont justiciables, si l'Etat n'a pas agi pour réaliser progressivement un droit dans un délai raisonnable. ${ }^{70}$

\section{\$1. Jugements sur les devoirs immédiats concernant le droit au logement}

Des tribunaux ont réussi à faire respecter le principe des devoirs immédiats, par exemple en ce qui concerne la protection judiciaire contre les expulsions forcées. Le droit au logement comprend le devoir positif de fournir un logement aux personnes dans le besoin, avec une réalisation progressive pendant un certain temps.

${ }^{60}$ [BVerfGE 45, $\left.187(229)\right]$.

${ }^{61}$ [BVerfGE 1, 97 (105)].

${ }^{62}$ [BVerfGE 40, $\left.121(133)\right]$.

63 Ibidem.

${ }^{64}$ Voir Tribunal fédéral suisse, V contre la Commune X et le Conseil d'Etat du canton de Berne, ATF 121 I 367 du 27 octobre 1995.

${ }^{65}$ Voir Tribunal suprême fédéral du Brésil (Supremo Tribunal Federal), RE 436996/SP (opinion écrite par le juge Celso de Mello), 26 octobre 2005.

66 Voir Cour suprême argentine, Reynoso, Nida Noemí c/ INSSJP s/amparo, 16 Mai 2006 (opinion majoritaire approuvant les arguments du procureur général).

${ }^{67}$ L'article 2 (1) se lit: «Chacun des Etats parties au présent Pacte s'engage à agir, tant par son effort propre ».

${ }^{68}$ Voir, dans le même sens, les Principes de Limbourg §§ 8, 16, 21-24.

${ }^{69}$ Voir, par exemple, Cour constitutionnelle colombienne, arrêt T-484/1992, 11 août 1992.

${ }^{70}$ Voir, par exemple, Cour constitutionnelle colombienne, arrêt T-595/2002, 1er août 2002, § 5.3.

DOI: 10.9790/0837-2109041329 www.iosrjournals.org


Mais l'Etat a aussi le devoir négatif immédiat de s'abstenir d'expulser des personnes de leur logement sans justification légale. Même quand elle se justifie, l'expulsion est interdite si elle ne s'accompagne pas de garanties de procédure. Ainsi, dans l'affaire $A S K c$. Bangladesh ${ }^{71}$ la Cour suprême de ce pays a affirmé qu'avant de procéder à l'expulsion massive des habitants d'une zone occupée sans autorisation, le Gouvernement doit élaborer un plan de rétablissement, effectuer les expulsions graduellement et tenir compte de la capacité des personnes expulsées de trouver un autre logement. Elle a ajouté que les autorités doivent donner un préavis suffisant avant l'expulsion. ${ }^{72}$

\section{\$2. Jugements sur les obligations immédiates concernant le droit au travail}

Le droit au travail nous donne aussi quelques exemples intéressants de la justiciabilité des obligations immédiates. Parmi ces dernières figurent l'interdiction de la discrimination, l'interdiction du travail forcé, le versement d'une rémunération équitable et la création des conditions de travail compatibles avec la dignité humaine. La Commission africaine des droits de l'homme et des peuples a considéré que l'Etat partie, quelles que soit ses ressources, a l'obligation de déceler et d'éradiquer des pratiques qui violent les droits inscrits dans la Charte africaine. Dans une décision remarquée touchant un pays à bas revenu (la Mauritanie), elle a déclaré à propos d'allégations d'esclavage à grande échelle:

"Indépendamment de la justification donnée par l'Etat défendeur, la Commission considère à la suite des dispositions de l'article 23(3) de la Déclaration universelle des droits de l'Homme que, quiconque travaille a droit à une rémunération équitable et satisfaisante lui assurant, ainsi qu'à sa famille, une existence conforme à la dignité humaine et complétée s'il y a lieu, par tous autres moyens de protection sociale. Ces dispositions sont complétées par celles de l'article 7 du Pacte international relatif aux droits économiques, sociaux et culturels. Eu égard à ce qui précède, la Commission retient une violation de l'article 5 de la Charte pour pratiques analogues à l'esclavage et souligne qu'un travail sans compensation équivaut à la violation du droit au respect de la dignité inhérente à la personne humaine. En outre, elle considère par ailleurs que les conditions auxquelles sont soumis les descendants d'esclaves constituent une exploitation et un avilissement de l'homme; toutes pratiques condamnées par la Charte africaine». ${ }^{73}$

La Cour interaméricaine des droits de l'homme s'est prononcé de la même manière dans l'affaire des massacres d'Ituango $^{74}$, qui porte, entre autres, sur des violations du droit au travail. A la suite du massacre des civils commis par des groupes paramilitaires avec la complicité de membres de l'armée colombienne, les coupables volent le bétail des victimes et forcent dix-sept paysans à amener, sans rémunération, les animaux sur le territoire en leur possession. La Cour a considéré que l'interdiction du travail forcé s'applique directement et a invoqué le paragraphe 2 de l'article 6 (interdiction de travail forcé ou obligatoire) et l'article 7 (droit à la liberté de la personne) de la Convention américaine relative aux droits de l'homme, ainsi que la Convention $n^{\circ} 29$ de l'Organisation internationale du travail, pour conclure que l'Etat est responsable de l'infraction.

\section{Section 2 : Approche juridictionnelle à propos de l'interdiction des mesures régressives}

La Commission des droits économiques, sociaux et culturels (CDESC) a étudié l'interdiction faite aux Etats d'adopter délibérément des mesures régressives ${ }^{75}$, ce qui correspond à l'un des principes sur lesquels s'appuient les juges et les avocats dans plusieurs domaines. La doctrine fondamentale est que, tout en reconnaissant la nécessité d'avancer pas à pas, le Pacte international relatif aux droits économiques, sociaux et

${ }^{71}$ Voir Cour suprême du Bangladesh, Ain o Salish Kendra (ASK) v. Government and Bangladesh \& Ors 19 BLD (1999) 488, 29 juillet 2001.

72 Ibidem.

${ }^{73}$ Commission africaine des droits de l'homme et des peuples, Malawi African Association et autres $c$. Mauritanie, Communications 54/91, 61/91, 98/93, 164/97-196/97 et 210/98 (2000), 11 mai 2000, § 135.

${ }^{74}$ Voir Cour interaméricaine des droits de l'homme, Massacres d'Ituango c. Colombie, ler juillet 2006, $\$ 145-$ 168.

${ }^{75}$ Voir CDESC, Observation générale $n^{\circ}$ 3, La nature des obligations des Etats parties (5e session, 1990), Doc. ONU E/1991/23, § 9; Observation générale $n^{\circ} 13$, Le droit à l'éducation (21e session, 1999), Doc. ONU E/C.12/1999/10 (1999), § 45; Observation générale $\mathrm{n}^{\circ} 14$, Le droit au meilleur état de santé susceptible d'être atteint (22e session, 2000) Doc. ONU E/C.12/2000/4 (2000), § 32; Observation générale $n^{\circ} 15$, Le droit à l'eau (29e session, 2003), Doc. ONU E/C.12/2002/11 (2003), § 19; Observation générale $\mathrm{n}^{\circ} 17$, Le droit de chacun de bénéficier de la protection des intérêts moraux et matériels découlant de toute production scientifique, littéraire ou artistique dont il est l'auteur (article 15, § $1 \mathrm{c})$ du Pacte), $\left(35^{\mathrm{e}}\right.$ session, 2005) Doc. ONU E/C.12/GC/17 (2006), § 27; Observation générale $\mathrm{n}^{\circ} 18$, Le droit au travail, (35e session, 2006), Doc. ONU E/C.12/GC/18 (2006) § 21 ; voir aussi le point 14 e) des Principes de Maastricht.

DOI: 10.9790/0837-2109041329 www.iosrjournals.org $\quad 23 \mid$ Page


culturels exige que l'Etat agisse pour assurer progressivement le plein exercice de ces droits et ne prenne pas des dispositions pour en retarder ou en supprimer la jouissance.

Dans diverses juridictions, les tribunaux nationaux se fondent sur l'interdiction de régression en cas de contestations de la suppression des services de santé publics, de modification de la loi sur le revenu minimal, de problèmes concernant la santé et la sécurité des travailleurs, etc. Ainsi, le Tribunal constitutionnel du Portugal s'est penché sur la contestation de l'amendement d'une loi garantissant un revenu minimal ${ }^{76}$, élevant de 18 à 25 ans l'âge requis pour obtenir les prestations prévues, ce qui en privait des personnes qui en bénéficiaient auparavant. Il a déclaré, entre autres, qu'il s'agissait du droit constitutionnel à la sécurité sociale et que, par conséquent, la nouvelle loi réduisant le nombre de bénéficiaires, ce qui enlevait ce droit aux personnes appartenant à la catégorie exclue, était anticonstitutionnelle.

La Cour suprême de l'Argentine est parvenue à la même conclusion lors d'un recours contre une modification de la loi relative aux prestations concernant la santé et la sécurité des travailleurs. ${ }^{77}$

Ainsi, la Cour constitutionnelle de la Colombie a abrogé des lois régressives concernant les pensions ${ }^{78}$, 1 'accès aux soins de santé ${ }^{79}$, l'instruction publique ${ }^{80}$ et la protection de la famille et des travailleurs ${ }^{81}$, et des règlements administratifs touchant le logement. ${ }^{82}$ Toutefois, dans certains cas, la Cour a considéré que les justifications avancées par le gouvernement pour diminuer la protection des travailleurs contre les licenciements sont suffisantes pour surmonter les présomptions habituelles contre de telles mesures. ${ }^{83}$ De nombreux organes judiciaires veillent au respect des droits économiques, sociaux et culturels inscrits dans la constitution, y compris ceux qui concernent les soins de santé et les pensions, en vérifiant si les décisions de l'Etat sont raisonnables, pertinentes, et proportionnelles. Ainsi, dans l'affaire Asociación de Esclerosis Múltiple de Salta ${ }^{84}$, la Cour suprême de l'Argentine a maintenu le jugement du tribunal d'appel qui annule un règlement du ministère de la santé excluant certains traitements de la sclérose en plaques du plan d'assurance maladie minimale obligatoire. La Cour a suivi l'avis du procureur général qui a considéré que le règlement est déraisonnable parce qu'il affecte le droit à la santé protégé par les traités internationaux relatifs aux droits de l'homme. Selon le procureur, l'Etat n'apporte pas de justification raisonnable pour expliquer la suppression de la couverture médicale totale pour certains bénéficiaires antérieurs. ${ }^{85}$

La Cour constitutionnelle tchèque s'est prononcée dans le même sens ${ }^{86}$. Elle a supprimé des conditions à remplir obligatoirement pour obtenir une pension, les considérant inutiles, disproportionnées et contraires au principe de l'égalité. La loi exigeait que le bénéficiaire potentiel présentât une demande dans un délai de deux ans pour obtenir une pension pour enfant à charge ; et sur ce, la Cour a reconnu que l'Etat peut légitimement se fixer l'objectif de gérer correctement le financement public de la sécurité sociale, ce qui peut comporter la limitation de la période de demande de prestations, mais elle a estimé qu'il est possible d'atteindre cet objectif par d'autres moyens qui ne portent pas atteinte à un droit fondamental. ${ }^{87}$ De même, la Cour suprême des EtatsUnis a jugé que les conditions restrictives pour l'octroi de bons d'alimentation sont anticonstitutionnelles et confirme par là la décision d'un tribunal d'instance inférieure d'inclure les plaignants dans le programme d'aide aux plus démunis. ${ }^{88}$

\footnotetext{
${ }^{76}$ Tribunal constitutionnel du Portugal, arrêt (Acórdão) № 509/2002, 19 décembre 2002.

${ }^{77}$ Cour suprême de l'Argentine, Aquino, Isacio c. Cargo Servicios Industriales S.A. s/accidentes ley 9.688, 21 septembre 2004.

${ }^{78}$ Voir Cour constitutionnelle de la Colombie, décision T-789/2002, 24 septembre 2002.

${ }^{79}$ Voir Cour constitutionnelle de la Colombie, décision T-671/2002, 20 août 2002.

${ }^{80}$ Voir Cour constitutionnelle de la Colombie, décision C-991-2004, 12 octobre 2004.

${ }^{81}$ Voir Cour constitutionnelle de la Colombie, décision $T-1318 / 2005,14$ décembre 2005.

${ }^{82}$ Voir Cour constitutionnelle de la Colombie, décision C-931-2004, 29 septembre 2004.

${ }^{83}$ Voir, par exemple, Cour constitutionnelle de la Colombie, décision C-038/2004, 27 janvier 2004. La Cour pense que l'objectif fixé par l'Etat, la réduction du chômage, était impératif et que la nouvelle loi satisfait à certaines conditions: 1) l'examen attentif du projet par le parlement, 2) l'étude d'autres moyens et 3) la proportionnalité des mesures adoptées par rapport à l'objectif visé. Voir $\S \S 32-48$.

${ }^{84}$ Voir Cour suprême de l'Argentine, Asociación de Esclerosis Múltiple de Salta c. Ministerio de Salud-Estado Nacional s/acción de amparo-medida cautelar, mémoire du procureur du 4 août 2003, Arrêt de la Cour du 18 décembre 2003.

${ }^{85}$ Ibidem.

${ }^{86}$ Voir Cour constitutionnelle de la République tchèque, PLUS 42/04, 6 juin 2006.

${ }^{87}$ Ibidem.

${ }^{88}$ Voir Cour suprême des Etats-Unis, US Department of Agriculture v. Moreno, 413 US 528, 25 juin 1973.

DOI: 10.9790/0837-2109041329 www.iosrjournals.org $24 \mid$ Page
}


D'autres jugements concernant les droits économiques, sociaux et culturels se fondent sur la constitution ou la législation pour faire respecter des droits fondamentaux, comme un «logement convenable», un «traitement approprié» ou un «enseignement élémentaire suffisant». Plusieurs arrêts de Cours suprêmes des Etats des Etats-Unis portant sur le devoir de fournir une instruction publique de qualité minimale le démontrent. Par exemple, la Cour suprême de New York constate, dans l'affaire Campaign for Fiscal Equity v. State of New York, que le financement de l'instruction publique ne satisfait pas aux prescriptions constitutionnelles minimales en vertu desquelles l'Etat doit fournir un «bon enseignement primaire». ${ }^{89}$ L'essentiel de cet arrêt est confirmé en appel.

\section{Section 3 : Autres droits humains sociaux examinés dans la jurisprudence internationale}

Dans la présente section, force nous est de noter qu'il existe d'autres exemples pratiques des droits humains sociaux qui ont fait objets d'examen devant des les juges étrangers. En effet, sans toutefois être exhaustive, la liste inclue le droit à un logement convenable, le droit à la santé, le droit à la sécurité sociale, ainsi que le droit à l'emploi.

\section{§1. Le droit à un logement convenable}

Dans son arrêt, aujourd'hui célèbre, prononcé en 2001 dans l'affaire Grootboom ${ }^{90}$, la Cour constitutionnelle de l'Afrique du Sud a procédé selon une analyse sui generis, quand elle a évalué la constitutionnalité d'un programme de logements mis en œuvre par le gouvernement. Un groupe de personnes, expulsées peu auparavant par les autorités locales d'une zone d'habitat spontané à Oostenberg, près du Cap, a demandé à la Cour suprême d'obliger l'Etat à leur fournir un abri temporaire pendant qu'elles cherchent un logement permanent. La Cour a accepté, arguant que les enfants du groupe ont le droit d'être logés aux frais de l'Etat, en vertu de l'article 28 1.c) de la constitution sud-africaine. Leurs parents doivent également être logés, car séparer les enfants de leurs parents ne serait pas dans leur intérêt supérieur et serait contraire aux dispositions de l'article 28 qui prévoit que l'intérêt supérieur de l'enfant doit primer dans toute décision concernant des mineurs. ${ }^{91}$

Avant que la Cour constitutionnelle n'ait examiné le dossier, le problème de ce groupe de plaignants a été résolu, parce que l'Etat a accepté de lui fournir un abri temporaire approprié. Par conséquent, elle s'est bornée à étudier la question constitutionnelle fondamentale suivante: en général, l'Etat est-il obligé de fournir un abri temporaire aux personnes qui n'ont pas de logement? Invoquant le droit de chacun d'avoir accès à un logement convenable prévu par l'article 26.1), elle a affirmé que l'Etat doit adopter un plan global et réalisable pour s'acquitter de ses obligations en la matière. La Cour a précisé qu'en choisissant le moyen de s'acquitter de ces obligations, les autorités doivent considérer trois facteurs:

- la nécessité de prendre des mesures législatives et autres raisonnables,

- la nécessité de procéder à la réalisation progressive de ce droit, et

- la nécessité d'utiliser des ressources disponibles. ${ }^{92}$

Concernant le caractère raisonnable des mesures, la Cour a dit que l'Etat a le devoir légal d'établir au moins un plan d'action pour améliorer le sort des personnes sans aucun abri, comme l'était la communauté de Grootboom. Ainsi l'étude de la politique pratiquée alors par l'Etat en matière de logement montre que celui-ci s'est concentré sur la fourniture des logements de longue durée, appropriés et à bas loyer, sans tenir compte de besoins d'abri temporaire des personnes à la rue. La Cour a estimé que la politique de l'Etat relative au logement est déraisonnable, donc anticonstitutionnelle, puisqu'elle néglige les sans-abri. ${ }^{93}$

\section{§2. Le droit à la santé}

\footnotetext{
${ }^{89}$ Voir Cour suprême de New York, Campaign for Fiscal Equity v. State of New York et al. 710 N.Y.S. 2d 475, 9 janvier 2001; voir également Cour d'appel de New York, Campaign for Fiscal Equity v. State of New York et al. 100 N.Y.S. 2d 893, 26 juin 2003, Cour d'appel de New York, 1ère division, Campaign for Fiscal Equity v. State of New York, 2006 NYSipOP 02284, 23 mars 2006.

${ }^{90}$ Cour constitutionnelle de l'Afrique du Sud, The Government of the Republic of South Africa and others $v$. Irene Grootboom and others, 2001 (1) SA 46 (CC), 4 octobre 2000.

${ }^{91}$ Grootboom v. Oostenberg Municipality (2000) 3 BCLR 277 (C).

${ }^{92}$ Ibidem.

${ }^{93}$ Supra note 87. 
La Cour constitutionnelle de l'Afrique du Sud s'est prononcée dans une affaire importante concernant le droit à la santé. Dans l'affaire South African Minister of Health v. Treatment Action Campaign ${ }^{94}$, elle juge déraisonnable le refus, par les services de santé publics, de fournir un médicament dont il est prouvé qu'il réduit la transmission du VIH de la mère à l'enfant. Force est de noter que dans le cadre de la prévention de la transmission du VIH, la Cour a opté pour l'application du critère de la raisonnabilité. En somme, dans l'affaire, la Cour analyse la pertinence des efforts déployés par le gouvernement pour lutter contre le SIDA, en particulier pour prévenir la transmission du VIH de la mère à l'enfant au moment de la naissance ; et en appel, la Cour constitutionnelle confirme l'essentiel de cette décision. Elle considère que le refus du gouvernement de fournir de la Névirapine de manière générale et le manque de plan global de prévention de la transmission du VIH de la mère à l'enfant sont déraisonnables et violent le droit des mères en situation de pauvreté et de leurs nouveau-nés d'accéder aux services de santé, droit prévu à l'article 27 de la constitution sud-africaine. ${ }^{95}$ Vu les preuves produites, la Cour rejette les préoccupations exprimées par le gouvernement au sujet de l'innocuité et de l'efficacité de la Névirapine. Elle pense que les services de santé publics ont la capacité latente d'administrer correctement ce médicament et d'en surveiller l'utilisation et les effets. La Cour ordonne donc au gouvernement de fournir de la Névirapine à tous les établissements de santé publics où son utilisation est indiquée et d'élaborer un plan global pour prévenir la transmission du VIH de la mère à l'enfant. ${ }^{96}$

\section{§3. Le droit à la non-discrimination en matière de logement et de prestations de la sécurité sociale}

Dans l'affaire Mme L. R. et consorts $c$. Slovaquiee ${ }^{97}$, le Comité pour l'élimination de la discrimination raciale estime qu'un plan de logements visant à satisfaire les besoins de la population rom équivaut à une discrimination pour des motifs d'origine ethnique. Le Comité des droits de l'homme de l'ONU est également saisi d'affaires dans lesquelles il rappelle que l'égalité devant la loi et l'interdiction de la discrimination s'appliquent aux droits économiques, sociaux et culturels. Dans Zwaan de Vries c. Pays-Bas ${ }^{98}$, il exprime l'opinion que la loi néerlandaise sur la sécurité sociale qui prévoit des prestations de chômage est discriminatoire à l'encontre des femmes mariées, appelées à satisfaire, pour en bénéficier, à des conditions que les hommes mariés ne doivent pas remplir. Le comité pense que cette différence de traitement fondée sur le sexe est contraire à l'article 26 du Pacte international relatif aux droits civils et politiques. La Cour européenne des droits de l'homme, elle aussi, se prononce de la même manière dans des cas semblables, les prestations sociales étant protégées par le droit à la propriété inscrit dans le protocole $\mathrm{n}^{\circ} 1$ à la Convention européenne. ${ }^{99}$

\section{§4. Le droit à la non-discrimination des salariés au motif de la maladie du VIH/SIDA}

Le droit à l'emploi est sacré et ce dernier ne peut être aliéné sous quel motif que ce soit. Le droit international protège le travailleur. ${ }^{100}$ En effet, demander à un salarié s'il est atteint de l'infection par le VIH comme condition posée préalablement à l'accès à l'emploi est contraire aux normes internationales protectrices relatives aux droits humains. ${ }^{101}$ L'information relative à l'état de santé du salarié relève strictement du secret médical. L'employeur ou le cabinet de recrutement ne peut demander à un candidat à l'embauche que des renseignements présentant un lien direct et nécessaire avec l'emploi proposé ou les aptitudes professionnelles requises pour le poste. ${ }^{102} \mathrm{Il}$ faut noter que le principe de non discrimination en raison de l'état de santé consacré

${ }^{94}$ Voir Cour constitutionnelle de l'Afrique du Sud, South African Minister of Health v. Treatment Action Campaign, 2002 (5) SA 721, 5 juillet 2002.

${ }^{95}$ Voir la Constitution de la République Sud-africaine adoptée en 1996.

${ }^{96}$ Voir supra note 91.

97 Voir Comité pour l'élimination de la discrimination raciale, Mme I. R. et consorts c. Slovaquie, Communication $\mathrm{n}^{\circ} 31 / 2003,10$ mars 2005.

98 Voir Comité des droits de l'homme, Zwaan de Vries c. Pays-Bas, Communication 182/1984, 9 avril 1987. Voir également Broeks c. Pays-Bas, Communication 172/1984, 9 avril 1987.

99 Voir Cour européenne des droits de l'homme, Wessels-Bergevoel c. Pays-Bas, 4 juin 2002 (discrimination fondée sur le sexe concernant la durée des prestations sociales), §§ 46-55; Willis c. Royaume-Uni, 11 juin 2003.

${ }^{100}$ La Charte africaine des droits de l'homme et des peuples, en son article 15 déclare : «Toute personne a le droit de travailler dans des conditions équitables et satisfaisantes et de percevoir un salaire égal pour un travail égal ».

${ }^{101}$ Se référer à l'article 23 de la Déclaration universelle de droits de l'homme de 1948 et aux articles 6 et 7 du Pacte international relatif aux droits économiques, sociaux et culturels de 1966.

${ }^{102}$ Les Directives internationales concernant le VIH/SIDA et les droits de l'homme conclues à Genève, 23-25 septembre 1996, indiquent ce qui suit: « Le droit au travail implique le droit de toute personne d'avoir accès 
par l'article 7 de la Déclaration universelle des droits de l'homme interdit à l'employeur de rechercher ou de se faire communiquer des informations d'ordre médical. Seul le salarié peut décider de révéler son état de santé à ses collègues de travail et à son employeur, le VIH ne se transmettant pas dans le cadre des relations de travail, l'employeur n'a exclusivement aucun motif ni de proposer, ni d'exiger systématiquement un test de dépistage du VIH au titre de la prévention des maladies dangereuses pour l'entourage, autrement le travail.

Dans son arrêt rendu en l'an 2000, la Cour constitutionnelle sud-africaine a déclaré ce qui suit :

«HIV is transmitted through intimate contact involving the exchange of body fluid. Thus, sexual intercourse, receipt of or exposure to the blood, blood products, semen, tissues or organs of the infected person or transmission from an infected mother to her foetus or suckling child are known methods by which it can be transmitted. HIV has never been shown to be transmitted through intact skin or casual contact... with the advent of treatment, individuals are capable of living normal lives and they can perform an employment tasks for which they are otherwise qualified". 103

Forcer est de noter qu'au lieu de discriminer l'employé suite à sa maladie ou refuser définitivement l'accès à l'emploi aux individus, un soutien psychologique de la personne infectée est essentiel pour diminuer le mal-être souvent inhérent à la séropositivité. La Cour constitutionnelle sud-africaine l'a jugé en ces termes: "People who are living with HIV/AIDS must be treated with compassion and understanding. They must not be condemned to "economic death" by the denial of equal opportunity in employment". ${ }^{104}$ Les personnes touchées par le SIDA ou par toute autre maladie grave disposent des différents recours juridiques lorsqu'elles sont victimes de discrimination dans l'exercice de leur activité professionnelle. En effet, en rendant justiciable le droit à l'emploi; la Cour constitutionnelle sud-africaine se fonde sur les normes internationales protectrices qui interdisent les discriminations à l'égard des personnes malades quelle que soit leur maladie. Il est d'ailleurs admis que juridiquement le SIDA ne se distingue pas des autres maladies. ${ }^{105}$

\section{CONCLUSION GENERALE}

Le développement actuel de droits humains permet de donner aux droits sociaux une valeur égale à celle dont bénéficient les droits civils et politiques ; cela veut dire qu'il faudrait considérer les droits sociaux comme les droits qui comportent, de plusieurs manières, des devoirs précis et des mécanismes d'exécution semblables à ceux qui existent pour les droits civils et politiques. Il convient de dire que l'évolution internationale des principes et des règles fondamentaux du droit international des droits de l'homme qui, à son tour, a inspiré les contenus des constitutions modernes des Etats, montrent que les droits sociaux sont justiciables. L'abondance de la jurisprudence supranationale disponible aujourd'hui en matière de défense des droits sociaux, met en évidence la possibilité d'obtenir, pour ces droits, une protection judiciaire.

En définitive, l'idée que les droits sociaux ne sont pas justiciables ne serait pas juste. Il faudrait d'ailleurs comprendre que la principale obligation d'assurer le respect effectif de ces droits incombe aux organes du Pouvoir public et pas aux tribunaux. En effet, ces derniers n'interviendraient que postérieurement au cas où il y a violation, afin de sanctionner les auteurs. Alors, s'il n'y a pas moyen d'assurer l'exécution du respect des droits sociaux, ceux-ci resteront utopiques et sous-estimés comparativement aux droits civils et politiques.

Dans cette étude qui s'achève, le but était de montrer que les droits économiques, sociaux et culturels tels que reconnus par le Pacte des Nations Unies relatif aux droits économiques, sociaux et culturels ne sont pas seulement devenus justiciables avec le temps (évolution); ils le sont par nature. Les droits sociaux ne sont pas vagues ou ambigües comme certaines théories le disent. Leur contenu (obligations correspondantes des Etats) est aujourd'hui défini de manière assez précise. Il est possible et une nécessité à un organe judiciaire ou quasi judiciaire de contrôler le respect par l'Etat de ses engagements relatifs aux droits sociaux, sans toutefois porter atteinte au principe de la séparation des pouvoirs.

à l'emploi sans conditions autres que les capacités professionnelles requises. Ce droit est violé lorsqu'un candidat ou un salarié est tenu de subir un test obligatoire de dépistage du VIH et se voit refuser l'emploi ou le perd, ou se voit refuser des prestations dues aux salariés si le résultat est positif. Les Etats doivent veiller à ce que les personnes touchées par le VIH/SIDA soient autorisées à travailler aussi longtemps qu'elles sont capables de remplir les fonctions de leur emploi ».

${ }^{103}$ Hoffmann v. South African Airways (CCT17/00) 2000 ZACC 17; 2001 (1) SA 1; 2000 (11) BCLR 1235; 200012 BLLR 1365 (CC) (28 September 2000), paras 12, 14 (12).

${ }^{104}$ Hoffmann v. South African Airways (CCT 17/00) 2000 ZACC 17; 2001 (1) SA 1; 2000 (11) BCLR 1235; 200012 BLLR 1365 (CC) (28 September 2000), para. 38.

${ }^{105}$ Consultez Michel Miné, SIDA et droit du travail, disponible sur www.impatient@ medecinesdouces. com, consulté le 12 février 2012. 


\section{BIBLIOGRAPHIE}

A. Traités internationaux des droits de l'homme

- Charte des Nations Unies adoptée par l'Assemblée générale des Nations Unies en 1945.

- Convention relative aux droits de l'enfant de 1989.

- Déclaration et Plan d'action de Bangalore de 1995.

- Déclaration universelle des droits de l'homme de 1948.

- Pacte international relatif aux droits économiques, sociaux et culturels de 1966.

\section{B. Jurisprudence internationale}

- Comité des droits de l'homme, Zwaan de Vries c. Pays-Bas, Communication 182/1984, 9 avril 1987. Voir également Broeks c. Pays-Bas, Communication 172/1984, 9 avril 1987.

- Comité pour l'élimination de la discrimination raciale, Mme I. R. et consorts c. Slovaquie, Communication $\mathrm{n}^{\circ}$ 31/2003, 10 mars 2005.

- Commission africaine des droits de l'homme et des peuples, Malawi African Association et autres c. Mauritanie, Communications 54/91, 61/91, 98/93, 164/97-196/97 et 210/98 (2000), 11 mai 2000, § 135.

- Cour constitutionnelle colombienne, arrêt T-595/2002, $1^{\text {er }}$ août 2002, § 5.3.

- Cour constitutionnelle de l'Afrique du Sud, South African Minister of Health v. Treatment Action Campaign, 2002 (5) SA 721, 5 juillet 2002.

[1] Cour constitutionnelle de l'Afrique du Sud, The Government of the Republic of South Africa and others v. Irene Grootboom and others, 2001 (1) SA 46 (CC), 4 octobre 2000.

[2] Cour constitutionnelle de la Colombie, décision C-931-2004, 29 septembre 2004.

[3] Cour constitutionnelle de la Colombie, décision T-1318/2005, 14 décembre 2005.

[4] Cour constitutionnelle de la Colombie, décision T-789/2002, 24 septembre 2002.

[5] Cour d'appel de New York, Campaign for Fiscal Equity v. State of New York et al. 100 N.Y.S. 2d 893, 26 juin 2003, Cour d'appel de New York, 1ére division, Campaign for Fiscal Equity v. State of New York, 2006 NYSipOP 02284, 23 mars 2006.

[6] Cour européenne des droits de l'homme, Wessels-Bergevoel c. Pays-Bas, 4 juin 2002 (discrimination fondée sur le sexe concernant la durée des prestations sociales), §§ 46-55; Willis c. Royaume-Uni, 11 juin 2003.

[7] Cour interaméricaine des droits de l'homme, Massacres d'Ituango c. Colombie, 1er juillet 2006, §§ 145168.

[8] Cour suprême argentine, Reynoso, Nida Noemí c/ INSSJP s/amparo, 16 Mai 2006.

[9] Cour suprême de l'Argentine, Aquino, Isacio c. Cargo Servicios Industriales S.A. s/accidentes ley 9.688, 21 septembre 2004.

[10] Cour suprême de l'Argentine, Asociación de Esclerosis Múltiple de Salta c. Ministerio de Salud-Estado Nacional s/acción de amparo-medida cautelar, mémoire du procureur du 4 août 2003, Arrêt de la Cour du 18 décembre 2003.

[11] Cour suprême de New York, Campaign for Fiscal Equity v. State of New York et al. 710 N.Y.S. 2d 475, 9 janvier 2001.

[12] Cour suprême des Etats-Unis, US Department of Agriculture v. Moreno, 413 US 528, 25 juin 1973.

[13] Cour suprême du Bangladesh, Ain o Salish Kendra (ASK) v. Government and Bangladesh \& Ors 19 BLD (1999) 488, 29 juillet 2001.

[14] Grootboom v. Oostenberg Municipality (2000) 3 BCLR 277 (C).

[15] Hoffmann v. South African Airways (CCT 17/00) 2000 ZACC 17; 2001 (1) SA 1; 2000 (11) BCLR 1235; 200012 BLLR 1365 (CC) (28 September 2000), para. 38.

[16] Hoffmann v. South African Airways (CCT17/00) 2000 ZACC 17; 2001 (1) SA 1; 2000 (11) BCLR 1235; 200012 BLLR 1365 (CC) (28 September 2000), paras 12, 14 (12).

[17] Tribunal constitutionnel du Portugal, arrêt (Acórdão) N No 509/2002, 19 décembre 2002.

[18] Tribunal fédéral suisse, V contre la Commune X et le Conseil d'Etat du canton de Berne, ATF 121 I 367 du 27 octobre 1995.

[19] Tribunal suprême fédéral du Brésil (Supremo Tribunal Federal), RE 436996/SP, 26 octobre 2005.

\section{Ouvrages généraux}

[20] Azizur Rahman, Chowdhury et Jahid H. Bhuiyan, An Introduction to International Human Rights Law, Leiden: Martinus Nijhoff Publishers, 2010.

[21] Buergental, Thomas, International Human Rights, $2^{\text {nd }}$ edition, Washington: West Publishing Co, 1995. 
[22] Clapham, Andrew, Human Rights: a very short Introduction, Oxford: Oxford University Press, 2007.

[23] Commission Internationale des Juristes (CIJ), Les tribunaux et l'application des droits économiques, sociaux et culturels : étude comparative d'expériences en matière de justiciabilité, série des droits de l'homme et état de droit, No 2, Genève, 2010.

[24] Degni-Segui, R., Les droits de l'homme en Afrique noire francophone: théorie et réalités, Abidjan: Imprimob, 1997.

[25] Delmas-Marty, Mireille, Le relatif et l'universel, Paris : Seuil, 2004.

[26] Donnelly, Jack, Universal Human Rights in Theory and Practice, London: Cornell University Press, 1989.

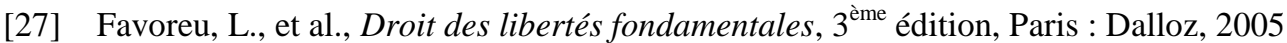

[28] Gregorio, P., Théorie générale de droits fondamentaux, Paris : LGDJ, 2004.

[29] Haas, Michael, International Human Rights: a comprehensive Introduction, London \& New York: Routledge, 2008.

[30] Janusz, Symonides, Human Rights: Concept and Standards, London: Ashgate Publishing Company, 2000.

[31] Leah, Levin, Droits de l'homme: questions et réponses, Paris : Editions UNESCO, 2005.

[32] Leiser, M. Burton, et Campbell, D. Tom, Human Rights in Philosophy and Practice, London: Ashgate Publishing Company, 2001.

[33] Levinet, M., Théorie générale des droits et libertés, Bruxelles: Bruylant, 2006.

[34] Mourgeon, Jacques, Les droits de l'homme, $1^{\text {ère }}$ édition, Paris : PUF, 1978.

[35] Patman, G. Robert, Universal Human Rights, London: Macmillan Press Ltd, 2000.

[36] Rehman, David, International Human Rights Law: a practical Approach, London: Longman, 2003.

[37] Rhona, K.M., et Smith, International Human Rights: Texts and Materials, London: Routledge \& Cavendish, 2007.

[38] Robertson, H., et Merrills, J. G., Human Rights in the World: an Introduction to the Study of the International Protection of Human Rights, $3^{\text {rd }}$ edition, Manchester: Manchester University Press, 1994.

[39] Rosenberg, G., The Hollow Hope: Can Courts Bring About Social Change?, Chicago: University of Chicago Press, 1991, pp 2 et ss.

[40] Tomuschat, Christian, Human Rights: between Idealism and Realism, New York: Oxford University Press, 2003.

[41] Wachsamann, P., Les droits de l'homme, $4^{\text {ème }}$ édition, Paris : Dalloz, 2002.

\section{Revues juridiques internationales}

[42] Jullien, «Universels, les droits de l'homme ? » in Le Monde Diplomatique, février 2008.

[43] Laïd Zaghlami, «Droits de l'homme et développement humain: des concepts qui ne font pas le consensus », in La Tribune (Alger), 25 juin 2005, p. 1.

[44] Marks, P. Stephen, "The Past and Future of the Separation of Human Rights into Categories", Maryland Journal of International Law, vol. 24, pp. 7-10.

[45] Neier, A., "Social and Economic Rights: A Critique", in Human Rights Brief 13-2 (2006), 1-3.

[46] Raffin, Marcello, « Pour une généalogie de la question des droits de l'homme » in Aspects, $\mathrm{n}^{\circ}$ 1, 2008.

[47] Robinson, Mary, “The Universal Declaration of Human Rights : a Living Document”, Australian Journal of International Affairs, Vol. 52, No. 2, juillet 1998, p. 118.

[48] Scott, C.; Macklem, P., "Constitutional Ropes of Sand or Justiciable Guarantees? Social rights in a new South African Constitution”, in University of Panslavania Law Review, vol. 144, No 1, 1992, p. 17.

[49] Tomasevski, K., «Justiciabilité des droits économiques, sociaux et culturels », in La Revue, Commission internationale des juristes, décembre 1995, No 55, p. 2.

[50] Tomuschat, C., "An Optional Protocol for the International Covenant on Economic, Social and Cultural Rights?”, in Weltinnenrecht-Liber amicorum Jost Delbrück, Duncker \& Humblot, Berlin, 2005, pp. 815834.

[51] Vasak, K., (1976): Le droit international des droits de l'homme, Recueil des cours de l'Académie de droit international, 1974-IV, La Haye : A.W.Sijthoff, Leyde ; pp. 388-389. 